\title{
Phase-shifting by means of an electronically tunable lens: quantitative phase imaging of biological specimens with digital holographic microscopy
}

\author{
Carlos Trujillo, ${ }^{1, *}$ Ana Doblas, ${ }^{2,3}$ Genaro SaAvedra, ${ }^{3}$ Manuel Martínez- \\ CORRAL, ${ }^{3}$ JORgE GARCíA-SUCERQUIA, ${ }^{1}$ \\ ${ }^{1}$ Universidad Nacional de Colombia, Sede Medellin, School of Physics, A.A. 3840 Medellin 050034, Colombia \\ ${ }^{2}$ Department of Physics and Astronomy, University of North Carolina at Chapel Hill, Chapel Hill, North Carolina 27599, USA \\ ${ }^{3} 3 \mathrm{D}$ Imaging and Display Laboratory, Department of Optics, University of Valencia, Burjassot, 46100, Spain \\ *Corresponding author: catrujila@unal.edu.co
}

Received XX Month XXXX; revised XX Month, XXXX; accepted XX Month XXXX; posted XX Month XXXX (Doc. ID XXXXX); published XX Month XXXX

The use of an electronically-tunable lens (ETL) to produce controlled phase shifts in interferometric arrangements is shown. The performance of the ETL as phase-shifting device is experimentally validated in phase-shifting digital holographic microscopy. Quantitative phase maps of a section of the thorax of a Drosophila melanogaster fly and of human red blood cells (RBCs) have been obtained using our proposal. The experimental results validate the possibility of using the ETL as a reliable phase shifter device. (C) 2015 Optical Society of America

OCIS codes: (050.5080) Phase-shift; (070.7345) Wave propagation; (090.1995) Digital holography; (100.5070) Phase retrieval.

http://dx.doi.org/10.1364/OL.99.099999

Phase shifting interferometry (PSI) is a numerical technique originally developed to recover the phase difference coded in interferometry intensity recordings [1]. PSI allows the pixel-wise recovering of the phase difference over a complete plane, producing what is known as a map of phase differences. Because such phase map accounts for the optical path difference between two ways the light waves have travelled, from its accurate computation wavelength sensitive displacements and/or deformations [2], pressure and/or temperature changes [3], index of refraction [4], among many other variables, can be precisely determined.

As the phase map represents the phase difference with respect to a well-known wave (reference wave), the same PSI algorithm can be utilized to compute the amplitude and phase of the unknown wave (object wave) over the recording plane of the interferogram. From that plane, the recovered complex amplitude can be numerically propagated to any other plane, which can include that where is located an object that introduces the phase difference. This method envisioned by Yamaguchi and Zhang [5], originated what is currently known as phase-shifting digital holography (PSDH). This technique, which is counted among those that allow the numerical reconstruction of digitally recorded holograms, has the remarkable feature of recovering the object wave without the zero diffraction order and the twin image, typically present in digital holography. This characteristic has made possible the utilization of PSDH for obtaining quantitative phase images (QPI) in in-line configurations to study surface shapes [6], the encryption of 3D information [7], the 3D object recognition [8], and so forth.

Despite the advance represented in the possibility of computing phase maps or even complex wavefields by means of PSI algorithms, the need of having several recorded interferograms with very well controlled phase shifts between them, could be the main drawback of this numerical method. Two approaches have been proposed to alleviate this disadvantage: i) the reduction of the needed phase shifts [9] and ii) the use of different experimental arrangements to introduce the required phase shifts [10]. Even though the number of required phase shifts can be reduced, in the best scenario at least two phase shifts are needed. Therefore new options to the production of well-controlled phase shifts are always welcome. In response to this need, we introduce in this letter a PSI method based on an electronically tunable lens (ETL) that shows a competitive performance in comparison with other PSI methods based on optoelectronic devices.

Different mechanical methods can be utilized for introducing the controlled phase shifts between the recorded interferograms. Perhaps the most utilized is based in mounting a reference optics on a piezoelectric transducer (PZT) [5,11]. This allows submicrometric movements to produce the needed phase shifts. Displacement of a plane mirror, rotation of a half-wave plate, translation of a diffraction grating or tilting of glass plate, are some of the options to produce the phase shift [1]. Although the mechanical-based systems are widely used, the main drawbacks of these methods are the difficulty of obtaining the perfect stability of the system, the robustness of the setups and the mechanical hysteresis they involve [1,12]. To avoid any mechanical movement and to alleviate the above-mentioned drawbacks, opto-electronically controlled devices, such as spatial light modulators (SLMs) and liquid crystal variable retarder/wave plate have been introduced in PSI systems to produce the needed phase shifts [13,14]. As opto-electronically controlled devices can be manipulated by a computer, automatic systems are easily configured, thus both the stability and the speed of these systems are greatly improved [15]. Among the opto-electronically controlled devices, the 
electronically tunable lenses (ETL) are increasingly utilized in optical research and technology because they can provide easy control of the optical power of imaging systems [16,17]. Although the use of the ETLs has extended to many applications, up to the best knowledge of the authors, this is the first proposal of its use as a PSI device. Notwithstanding the ETL operating as a PSI device can be utilized in any interferometric technique, we illustrate this method in digital holographic microscopy (DHM) $[14,18,19]$, as we produce quantitative phase images of two biological specimens.

In Fig. 1, a phase-shifting DHM (PSI-DHM) operating with an ETL as phase-shifting device is illustrated. The set-up is based on a modified Mach-Zenhder interferometer with the insertion of the ETL to operate as a PSI device. The light-beam emitted by a He-Ne laser with wavelength $\lambda=633 \mathrm{~nm}$ is spatial filtered and then collimated by the CL lens of focal length $f_{C L}=200 \mathrm{~mm}$. The resulting collimated beam impinges on a cube beam splitter (BS1) to create the object (0) and the reference (R) waves. At the sensor plane, the interference pattern between the latter waves, known as hologram, is recorded. At the recording plane, the object wave is produced by a regular microscopy imaging system that produces a magnified image of the specimen. To ease the phase imaging, the imaging system is composed by an infinitycorrected microscope objective (MO) and a tube lens (TL), operating in an optimized telecentric regime [20]. To numerically recover the complex object wave field scattered by the specimen, we choose the method proposed by De Nicola et al. [21] based on four phase shifted holograms. To introduce the controlled phase shifts needed in the recorded holograms, we propose the insertion of an ETL in the path of the reference wave. As shown in Fig. 1, the ETL is placed at the common focal plane of an afocal relay system composed by two converging lenses of focal lengths $f_{\mathrm{L} 1}=150 \mathrm{~mm}$ and $f_{\mathrm{L} 2}=200 \mathrm{~mm}$. These focal lengths have been chosen in order to match the size of the output beam of the relay system to completely illuminate the sensor area of the camera. The hallmark of the ETLs is the possibility of tuning its optical power by changing an applied voltage [22]. The change of optical power is achieved by modifying the geometry of a liquid drop, see Fig. 2. Such modification can be utilized to produce controlled phase shifts with great stability and accuracy.

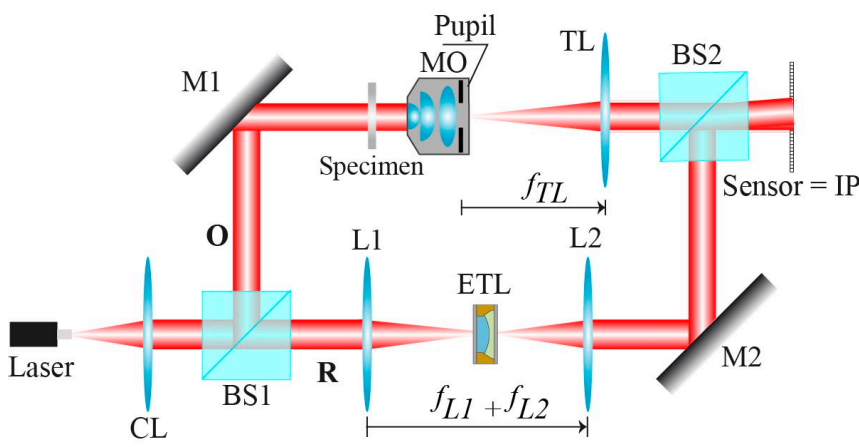

Fig. 1 Proposed set-up to provide PSI using an electronically tunable lens (ETL), which is inserted at the common focal plane of an afocal relay system. CL, collimating lens; BS1 and BS2, are cubes beamsplitters; 0 , object wave; $\mathrm{R}$, reference wave; $\mathrm{M} 1$ and $\mathrm{M} 2$, are mirrors; L1 and L2, converging lenses. The microscope objective MO and a tube lens TL operated in telecentric configuration.

To understand how a change in the voltage applied to the ETL introduces a phase-shift, we calculate the optical phase difference (OPD), without the specimen in place, between both interfering waves. The OPD at the sensor plane of Fig. 1 can be expressed as the difference between the optical path length of the reference wave, OPLR, and that of the object wave, OPLo,
To compute OPLR, note in Fig. 1 that the reference beam passes through three different optical elements: L1, L2, and ETL. In particular, the ETL is composed by two media whose individual thicknessesshape varies with the applied voltage by keeping constant the total thickness, $d_{E T L}=d_{C D}+d_{O i l}$. The radius of curvature of the oil phase is estimated to be between $31,8 \mathrm{~mm}$ and $37 \mathrm{~mm}$ for the range of voltages applied to the ETL. As the sample is not in place, the $O P L_{O}$ can be

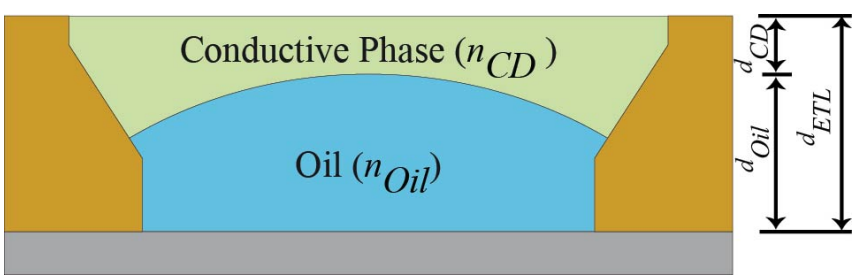

Fig. 2 Electronically tunable lens (ETL) schematics. In green, the conductive phase with refraction index nCD and in blue the oil phase with refraction index $n_{L L}$ dETL is the total thickness of the ETL, doil and $\mathrm{d}_{\mathrm{CD}}$ are thicknesses at the center of the ETL.

considered as a constant bias in the general $O P D$ term. With these considerations and after some algebra, Eq. (1) can be written as:

$$
O P D=C+\left(n_{C D}-n_{O i l}\right) d_{C D}(V),
$$

where $n_{C D}$ and $n_{O i l}$ are, the refraction indexes of the conductive and oil phases, respectively (see Fig. 2). Besides,

$$
C=\left(n_{L 1}-1\right) d_{L 1}+\left(n_{L 2}-1\right) d_{L 2}+n_{O i l} d_{E T L}
$$

is a constant that depends on the refractive indexes and the thicknesses of the optical elements inserted in the reference arm. Finally $d_{C D}(V)$ is the thickness of the conductive phase, which can be modified by applying different voltages to the ETL. As a result, a voltage variation of the ETL introduces a change in the OPD, which is translated into a phase shift by

$$
\Delta \varphi=\frac{2 \pi}{\lambda} O P D,
$$

with $\lambda$ the illumination wavelength. Eq. (4) confirms that ETLs can be used to generate phase-shifts, when using their central region. Despite the fact that the above calculation of the OPD is performed for the optical axis, the use of a relay system operating in telecentric regime allows its extension to the whole field of illumination, see Fig. 1. The latter assumption is valid if the focused beam can be considered to be a point at the axial point of the diopter that separates the two media in the ETL. According to Siegman [23], for the case of the lens L1, the spot diameter is about $7.5 \mu \mathrm{m}$. This is considerably smaller than the diopter diameter. Thus, we can ignore the curvature of the diopter and consider all the thicknesses mentioned in Eq. (2) and (3) to be not functions of $x$ and $y$, that is, the lateral coordinates. Other parameter of the focused beam is the Rayleigh range, which is this case was $\mathrm{Z}_{\mathrm{R}}=70$ $\mu \mathrm{m}$. This range covers by large the shift suffered by the axial point of the diopter in the whole experiment.

$$
O P D=O P L_{R}-O P L_{O}
$$



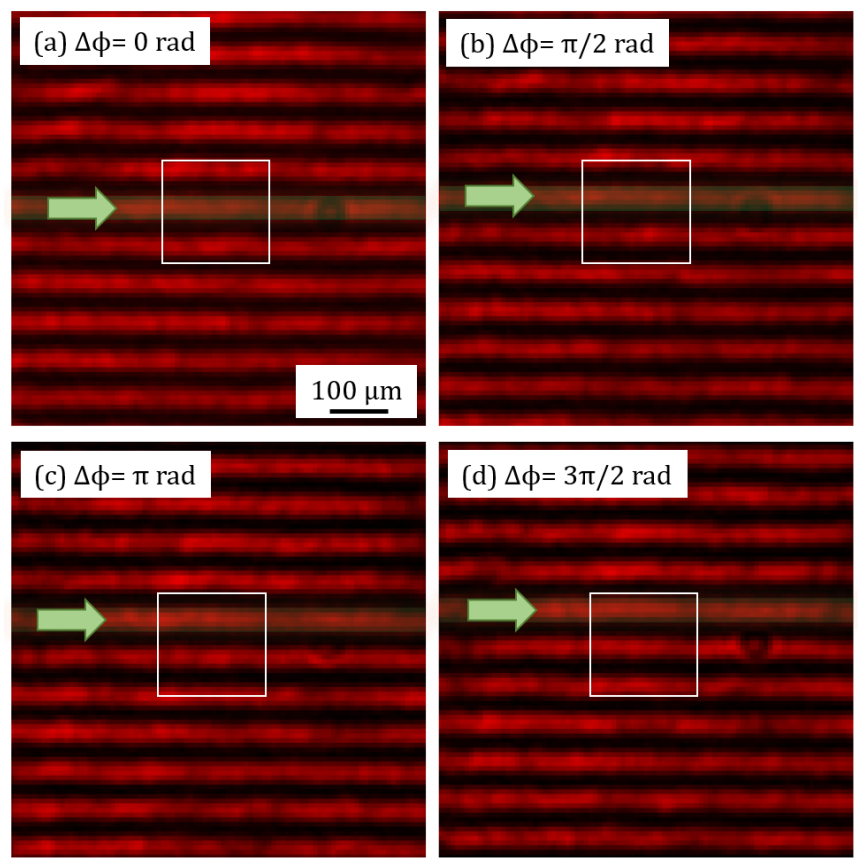

Fig. 3 Experimental holograms as an ETL is used as phase-shifting device. The arrow marks a given fringe as it is moved in regard with the fixed square.

To confirm the use of the ETL as a phase-shifting device, we recorded a set of holograms after tuning the voltage of the ETL, see Fig. 3. For this experiment we used a CCD camera of $1224 \times 1024$ square pixels (6.9 $\mu \mathrm{m}$ width) the liquid lens ARTIC 39N0 (VARIOPTIC) [22]. The ARTIC 39N0 has a nominal optical aperture of $\phi_{P T L L}=3.5 \mathrm{~mm}, \mathrm{a}$ maximum wavefront error of $50 \mathrm{~nm}$ and transmission of $97 \%$ at $\lambda=587 \mathrm{~nm}$. According to manufacturer's datasheet, the relation between the applied voltage and the thickness of the conductive phase is

$$
d_{C D}(V)=-0.0138 V+1.8764 \text {. }
$$

Even though a voltage is applied to the ETL, according to the manufacturer's datasheet the range of the consumed current by the ETL is in the order of microamperes, thus any heating is negligible.

As shown in Fig. 3, each hologram is phase-shifted with respect to the others. From these data we found that a voltage change of $0.5 \mathrm{~V}$ produce a phase shift of $2 \pi$. Therefore we could state that $\pi / 2$ shifts can be obtained with voltage steps of $0.125 \mathrm{~V}$. The shown holograms are average images resulting from 20 shots at every voltage. The correlation parameter between those 20 images in each case is above $0.98 \pm 0.02$ (mean \pm std). As this value is close to 1 , we can conclude that the PSI system implemented with the ETL is highly stable and accurate. (a)
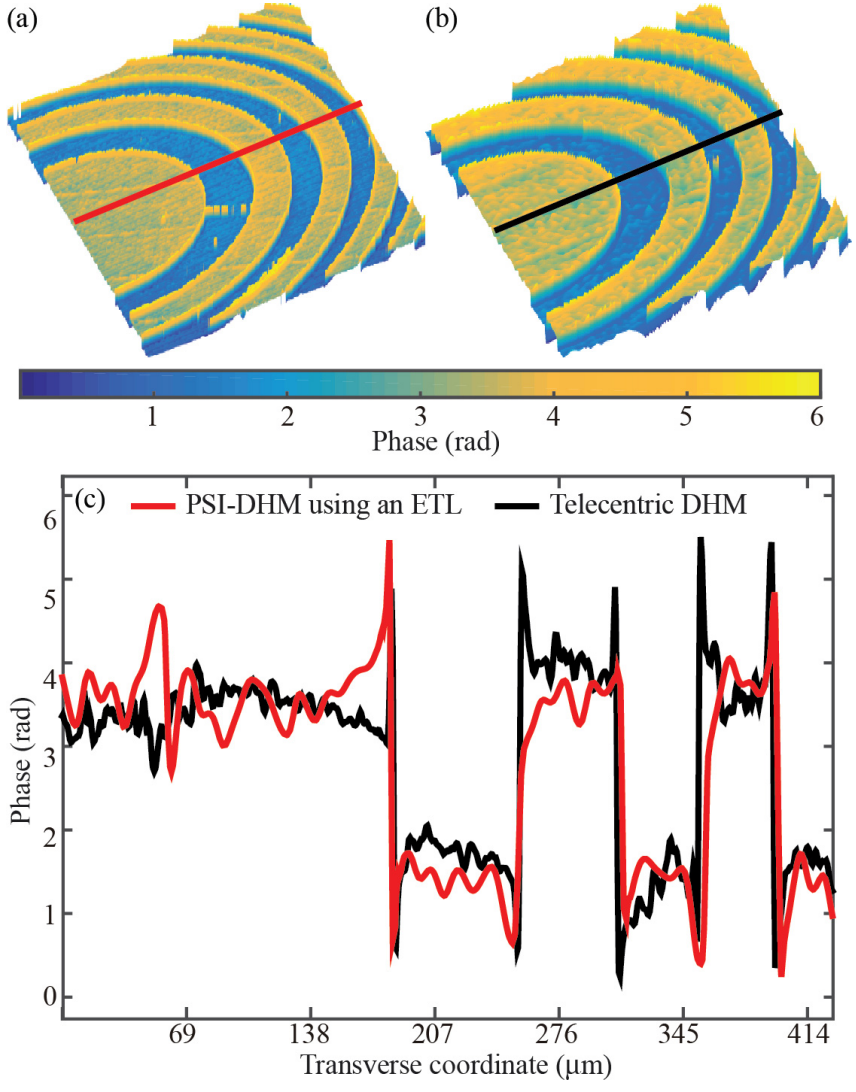

Fig. 4. PSI-DHM v.s. Off-axis telecentric DHM. (a) QPI of a Fresnel's lens by PSI-DHM; (b) QPI of the same Fresnel's lens by off-axis telecentric DHM and (c) profiles along the lines of panels (a) and (b).

The accuracy of the ETL as a phase-shifting device was tested by contrasting QPI measurements by a PSI-DHM operating with an ETL as phase-shifting device and by an off-axis optimized telecentric DHM [20]. In panel (a)/(b) of Fig. 4, the QPI map produced by PSIDHM/off-axis telcentric DHM of a calibrated Fresnel's lens is shown. According with the color bar, both methods report the same values of phase for the target. This observation is confirmed in panel (c), where profiles along the lines over the QPI maps are shown. The good agreement between the phase measurements reported by both methods constitute a proof of the accuracy of the measurements performed with PSI-DHM as using an ETL as phase shifting device.

Once the accuracy of the ETL as a PSI device has been tested, we performed a series of proof-of-concept experiments in which biological samples are imaged by using a PSI-DHM operating with an ETL as phase-shifting device as that illustrated in Fig. 1. As a first experiment, we image a section of the thorax of a Drosophila melanogaster fly. In this case, the imaging system is composed by a 10x/0.45 MO and a tube lens of $200 \mathrm{~mm}$ focal length. In Fig. 5, we show the recovered phase map of the sample after applying the proper reconstruction algorithm [21]. Note that details of the internal structure of the section of the thorax of the Drosophila melanogaster fly are clearly visible. In fact, we can identify: (1) the digestive tract, (2) the bundle of muscle cells responsible for the flight, (3) a few bundles of muscle cells transversally cut and (4) the longitudinal section of a bundle of muscle cells. 


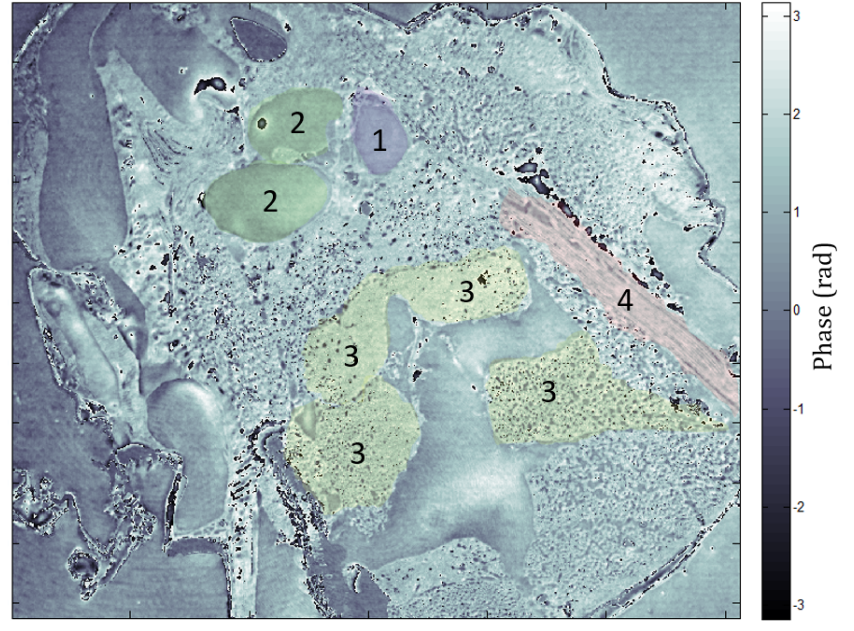

Fig. 5 QPI of a section of the thorax of a Drosophila melanogaster fly via PSI-DHM by using ETL as phase shifting device. Some parts of the image are labeled and pseudo colored for better visualization. See text for further details.

As a second experiment, we image human red blood cells (RBCs). The sample is prepared by smearing a blood drop on a glass slide. The smeared blood is dried out at room temperature in a dust-free environment. For this case, a 50x/0.55 MO is used. The recovered phase image of the human RBCs is shown in Fig. 6. Note that in Fig. 6 the measured phase has been converted into the optical thickness of the specimen. This is done by considering that the measured phase is related with the thickness of the sample $t$, the refractive index of the sample $n_{s}$, and the surrounding $n_{m}$ via $\phi=2 \pi / \lambda\left(n_{s}-n_{m}\right)$; for this experiment $n_{s}=1.406$ [24], $n_{m}=1$ and $\lambda=633 \mathrm{~nm}$.

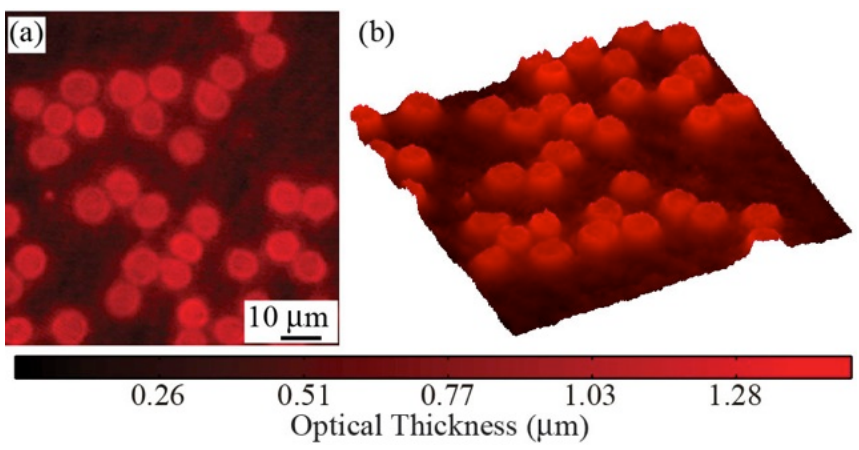

Fig. 6 QPI of human RBCs via PSI-DHM by using ETL as phase shifting device. Panel (a) is the recovered image in terms of optical thickness and panel (b) is its 3D view.

In summary, in this letter we have presented the use of an ETL as an accurate phase-shifting device. The main advantage is that the phase shifting is obtained avoiding any mechanical movements. Using the ETL ARTIC 39N0, we can obtain phase-shifts of $2 \pi / 5$ rad every 100 $\mathrm{mV}$, which allows a precise setting of the needed phase shifts for the implementation of any phase recovering via PSI. Even though the method can be utilized in any interferometry essay, the performance of the ETL as phase-shifting device has been experimentally validated in phase shifting DHM (PSI-DHM). Specifically, we have imaged a section of the thorax of a Drosophila melanogaster fly and human RBCs. Our experimental results show a great performance over the whole FOV, providing QPIs of great quality and accuracy for analyzing biological samples.

Funding This research was supported by the Spanish Ministry of the Economy and Competitiveness (DPI2012-32994) and also by the Autonomous Government of Valencia, Spain
(PROMETEOII/2014/072). C. Trujillo and A. Doblas acknowledge funding from the University of Valencia. J. Garcia-Sucerquia and C. Trujillo gratefully acknowledge the support from the youth research program, Colciencias-Universidad Nacional de Colombia (Hermes 28751)

\section{References}

1. K. Creath, Prog. Opt. 26, 349-393 (1988).

2. M. Chang, C.-P. Hu, P. Lam, and J. C. Wyant, Appl. Opt. 24, 3780 (1985).

3. E. Shoji, A. Komiya, J. Okajima, H. Kawamura, and S. Maruyama, Appl. Opt. 54, 6297 (2015).

4. E. W. Rogala and H. H. Barrett, J. Opt. Soc. Am. A 15, 538 (1998).

5. I. Yamaguchi and T. Zhang, Opt. Lett. 22, 1268 (1997).

6. I. Yamaguchi, T. Ida, M. Yokota, and K. Yamashita, Appl. Opt. 45, 7610-7616 (2006).

7. E. Tajahuerce and B. Javidi, Appl. Opt. 39, 6595-6601 (2000).

8. E. Tajahuerce, O. Matoba, and B. Javidi, Appl. Opt. 40, 3877 (2001).

9. Y. Awatsuji, T. Tahara, A. Kaneko, T. Koyama, K. Nishio, S. Ura, T. Kubota, and O. Matoba, Appl. Opt. 47, D183 (2008).

10. C. Robledo-Sanchez, R. Juarez-Salazar, C. Meneses-Fabian, F. Guerrero-Sánchez, L. M. Arévalo Aguilar, G. Rodriguez-Zurita, and V. Ixba-Santos, Opt. Express 21, 17228 (2013).

11. D. Malacara, M. Servín, and Z. Malacara, Interferogram Analysis For Optical Testing, (2005).

12. Y.-J. Lin and C.-L. Pan, Appl. Opt. 30, 1648 (1991).

13. Y. Bitou, Opt. Lett. 28, 1576 (2003)

14. G. Popescu, Quantitative Phase Imaging of Cells and Tissues (McGraw-Hill, 2012), Vol. 17.

15. C.-S. Guo, Z.-Y. Rong, H.-T. Wang, Y. Wang, and L. Z. Cai, Appl. Opt. 42, 6975-6979 (2003).

C.-J. Kim, M. Chang, M. Lee, J. Kim, and Y. H. Won, Appl. Opt. 54, 2565 (2015).

17. A. Doblas, D. Hincapie-Zuluaga, G. Saavedra, M. Martínez-Corral, and J. Garcia-Sucerquia, Appl. Opt. 54, 5229-5233 (2015).

T. Kreis, Handbook of Holographic Interferometry: Optical and Digital Methods (Wiley-vch Verlag Ed, 2005).

M. K. Kim, Digital Holographic Microscopy (Springer, 2011).

E. Sánchez-Ortiga, A. Doblas, G. Saavedra, M. Martínez-Corral, and J. Garcia-Sucerquia, Appl. Opt. 53, 2058-2066 (2014).

21. S. De Nicola, P. Ferraro, A. Finizio, and G. Pierattini, Opt. Lasers Eng. 37, 331-340 (2002).

22. B. Berge and J. Peseux, Eur. Phys. J. E 3, 159-163 (2000)

23.

A. E. Siegman, Lasers (University Science Books, 1986).

24. J. He, A. Karlsson, J. Swartling, and S. Andersson-Engels, J. Opt. Soc. Am. A 21, 1953 (2004). 


\section{Complete References}

[1] K. Creath, "Phase-Measurement Interferometry Techniques," Prog. Opt., vol. 26, no. C, pp. 349-393, 1988.

[2] M. Chang, C.-P. Hu, P. Lam, and J. C. Wyant, "High precision deformation measurement by digital phase shifting holographic interferometry," Appl. Opt., vol. 24, no. 22, p. 3780, Nov. 1985.

[3] E. Shoji, A. Komiya, J. Okajima, H. Kawamura, and S. Maruyama, "High-speed phase-shifting interferometry using triangular prism for time-resolved temperature measurement," Appl. Opt., vol. 54, no. 20, p. 6297, Jul. 2015.

[4] E. W. Rogala and H. H. Barrett, "Phase-shifting interferometer/ellipsometer capable of measuring the complex index of refraction and the surface profile of a test surface," J. Opt. Soc. Am. A, vol. 15, no. 2, p. 538, 1998.

[5] I. Yamaguchi and T. Zhang, "Phase-shifting digital holography," Opt. Lett., vol. 22, no. 16, p. 1268, Aug. 1997.

[6] I. Yamaguchi, T. Ida, M. Yokota, and K. Yamashita, "Surface shape measurement by phase-shifting digital holography with a wavelength shift," Appl. Opt., vol. 45, no. 29, pp. 76107616, 2006.

[7] E. Tajahuerce and B. Javidi, "Encrypting threedimensional information with digital holography.," Appl. Opt., vol. 39, no. 35, pp. 6595-6601, 2000.

[8] E. Tajahuerce, O. Matoba, and B. Javidi, "Shift-Invariant Three-Dimensional Object Recognition by Means of Digital Holography," Appl. Opt., vol. 40, no. 23, p. 3877, 2001.

[9] Y. Awatsuji, T. Tahara, A. Kaneko, T. Koyama, K. Nishio, S. Ura, T. Kubota, and O. Matoba, "Parallel two-step phase-shifting digital holography," Appl. Opt., vol. 47, no. 19, p. D183, Jul. 2008.

[10] C. Robledo-Sanchez, R. Juarez-Salazar, C. MenesesFabian, F. Guerrero-Sánchez, L. M. Arévalo Aguilar, G. RodriguezZurita, and V. Ixba-Santos, "Phase-shifting interferometry based on the lateral displacement of the light source," Opt. Express, vol. 21, no. 14, p. 17228, Jul. 2013.

[11] D. Malacara, M. Servín, and Z. Malacara, Interferogram Analysis For Optical Testing,. 2005.

[12] Y.-J. Lin and C.-L. Pan, "Precision displacement measurement by active laser heterodyne interferometry," Appl. Opt., vol. 30, no. 13, p. 1648, May 1991.

[13] Y. Bitou, "Digital phase-shifting interferometer with an electrically addressed liquid-crystal spatial light modulator," Opt. Lett., vol. 28, no. 17, p. 1576, Sep. 2003.

[14] G. Popescu, Quantitative Phase Imaging of Cells and Tissues, vol. 17, no. 2. McGraw-Hill, 2012.

[15] C.-S. Guo, Z.-Y. Rong, H.-T. Wang, Y. Wang, and L. Z. Cai, "Phase-shifting with computer-generated holograms written on a spatial light modulator.," Appl. Opt., vol. 42, no. 35, pp. 69756979, 2003.

[16] C.-J. Kim, M. Chang, M. Lee, J. Kim, and Y. H. Won, "Depth plane adaptive integral imaging using a varifocal liquid lens array," Appl. Opt., vol. 54, no. 10, p. 2565, Apr. 2015.

[17] A. Doblas, D. Hincapie-Zuluaga, G. Saavedra, M. Martínez-Corral, and J. Garcia-Sucerquia, "Physical compensation of phase curvature in digital holographic microscopy by use of programmable liquid lens," Appl. Opt., vol. 54, no. 16, pp. 52295233, 2015.

[18] T. Kreis, Handbook of Holographic Interferometry: Optical and Digital Methods. Weinheim: Wiley-vch Verlag Ed, 2005.

[19] M. K. Kim, Digital Holographic Microscopy. Springer, 2011.

[20] E. Sánchez-Ortiga, A. Doblas, G. Saavedra, M. MartínezCorral, and J. Garcia-Sucerquia, "Off-axis digital holographic microscopy: practical design parameters for operating at diffraction limit," Appl. Opt., vol. 53, no. 10, pp. 2058-2066, 2014.

[21] S. De Nicola, P. Ferraro, A. Finizio, and G. Pierattini, "Wave front reconstruction of Fresnel off-axis holograms with compensation of aberrations by means of phase-shifting digital holography," Optics and Lasers in Engineering, vol. 37, no. 4. pp. 331-340, 2002.

[22] B. Berge and J. Peseux, "Variable focal lens controlled by an external voltage: An application of electrowetting," Eur. Phys. J. E, vol. 3, no. 2, pp. 159-163, 2000.

[23] A. E. Siegman, Lasers. Mill Valley, California: University Science Books, 1986.

[24] J. He, A. Karlsson, J. Swartling, and S. Andersson-Engels, "Light scattering by multiple red blood cells," J. Opt. Soc. Am. A, vol. 21, no. 10, p. 1953, Oct. 2004. 